\section{Kawasaki syndrome}

In 1944 Scott and Rotondo reported the case of a 9 month old American girl who died suddenly of myocardial ischaemia and coronary aneurysms four weeks after developing fever, pharyngitis, pyuria, and a rash. ${ }^{1}$ This condition, then called infantile periarteritis nodosa, is pathologically identical with the syndrome later recognised by Kawasaki in Japan. ${ }^{2}$ Its cause remains unknown and the diagnosis is made from the characteristic clinical features. Eighty per cent of those affected are aged under 5 years. The first signs are fever and a generalised, often urticarial or morbilliform rash. Fever persists for at least five days and sometimes several weeks. Pharyngitis, cracking of the lips, prominence of the papillas of the tongue, and a non-exudative conjunctivitis develop within three days. The hands and feet become swollen and indurated with striking redness of the palms and soles. After two weeks the skin of the fingers and toes peels, starting at the tips adjacent to the nails. These signs occur in nearly all cases. Other features include, in order of frequency, a mass of enlarged lymph nodes in one side of the neck, urethritis, arthritis, disturbance of consciousness, aseptic meningitis, abdominal pain, hepatitis, and occasionally acute hydrops of the gall bladder. There is a neutrophil leucocytosis and a rise in the erythrocyte sedimentation rate and platelet count. Three quarters of the patients show a transient rise in serum concentrations of $\operatorname{IgE}$, but the value bears no relation to the severity of the disease. ${ }^{3}$ Autoantibodies are not present, and total haemolytic complement remains normal. ${ }^{4}$

One fifth of patients have clinical signs of heart disease. These include heart block, atrial arrhythmias, pericardial effusion, and mitral insufficiency, and by the fourth week coronary artery aneurysms. ${ }^{56}$ Death during the early acute phase is due mainly to myocarditis and inflammation of the conducting system; death between 12 and 25 days may be due to these lesions or to myocardial ischaemia and coronary aneurysms; and late deaths are due to ischaemic heart disease. ${ }^{7}$ The necropsy findings are of widespread vasculitis always affecting the coronary arteries.

Is the Kawasaki syndrome due to an infection? Five outbreaks of epidemic proportions, one in Japan and four in the United States, suggest that it might be. But in each outbreak there was no evidence of person to person transmission, no infective agent has been isolated consistently from affected children, and antibody studies have been inconclusive. A recent survey in the United States showed a seasonal variation in incidence, with clustering of cases between February and May. ${ }^{8}$ This might suggest some exogenous agent which prevails at that time of year. The survey confirmed the higher incidence in Asians than in whites and also found a higher incidence in black than in white children. At present we cannot say whether this reflects varying genetic susceptibility (there is no obvious association with HLA type ${ }^{9}$ ) or differing environmental circumstances.

The syndrome has become more common in Japan in the past 20 years as changes in living habits have led to a rise in the numbers of house dust mites in Japanese homes. ${ }^{10}$ Furusho et al found high concentrations of antimite IgG in 15 and antimite IgE in six of 20 children with the Kawasaki syndrome, ${ }^{11}$ and immune complexes containing mite antigen have been detected in patients' sera during the acute phase of their illness. ${ }^{12}$ If house mites play some part in the pathogenesis the mechanism might be an immune response to dermatophagoides antigens; alternatively the mite could be a vector for some infectious agent. Some rickettsia like bodies identified in the digestive canal of mites ${ }^{13}$ resemble particles seen in the lymph nodes, spleen, and skin of some patients with the Kawasaki syndrome. ${ }^{14}{ }^{15} \mathrm{~A}$ transient rise in exposure to mites may explain the strange association with the use of rug shampoo found in a recent outbreak of the syndrome in Colorado. ${ }^{16}$

The electrocardiogram is often abnormal, with ST-T wave changes and prolongation of the PR and QT intervals, but it gives no help in recognising coronary aneurysms. In one survey the electrocardiogram was normal in eight of 12 patients with aneurysms. ${ }^{8}$ A recent review suggested that echocardiograms should be done on all patients at two to three weeks and again at four to six weeks; the first examination would help assess myocardial and mitral valve function and look for pericardial effusions and the second help identify coronary aneurysms. ${ }^{3}$ Angiography is a little more sensitive for visualising aneurysms but more hazardous to perform. In a few instances aneurysms have been treated by aortocoronary bypass grafting, but their natural tendency is to regress, and serial echocardiograms have shown that over half resolve within a year. ${ }^{6}$

Definitive treatment must await an understanding of the syndrome's aetiology; but meanwhile patients should be monitored for the development of cardiac complications, and treatment with anti-inflammatory drugs may seem rational. Aspirin is potentially useful because it inhibits platelet aggregation as well as being an anti-inflammatory drug. In doses of $80-120 \mathrm{mg} / \mathrm{kg}$ daily it shortens the period of acute fever. ${ }^{17}$ Malabsorption of salicylates occurs in some patients, ${ }^{18}$ so blood concentrations should be monitored. Once the fever has settled a small dose (10 $\mathrm{mg} / \mathrm{kg}$ daily) should, perhaps, be continued for several weeks. The peak period of mortality (10-30 days) coincides with the time when the platelet count is highest. During the illness platelet aggregation is increased ${ }^{19}$ and synthesis of thromboxane $\mathrm{A}_{2}$ by the patient's platelets is increased. ${ }^{20}$ This metabolite of arachidonic acid is believed to provoke reactions between platelets and vessel walls in diabetic patients ${ }^{21}$ and may have a similar effect in Kawasaki syndrome. Small doses of aspirin appear to reduce platelet aggregation by inhibiting thromboxane $A_{2},{ }^{22}$ and Sasaguri and Kato have shown that there is less vascular damage in patients treated with small doses of aspirin. ${ }^{23}$ Corticosteroids appear to be contraindicated. They may increase the platelet count, and in one controlled study the incidence of coronary aneurysms was $65 \%$ in patients given steroids compared with $20 \%$ in those given no anti-inflammatory drugs and $11 \%$ in those given aspirin. ${ }^{5}$

The pathological features of the fatal disease are quite well documented, but we know very little about the long term outcome for those less severely affected. The mechanism of the spontaneous resolution of the aneurysms is not understood, but it seems unlikely that the coronary vessels return completely to normal. Only by careful follow up will we learn whether the syndrome predisposes to arteriosclerosis in later life.

JOHN PRICE

Consultant Paediatrician,

King's College Hospital,

London SE5 9RS

${ }^{1}$ Scott EP, Rotondo CC. Periarteritis nodosa; report of two cases, one complicated by intrapericardial hemorrhage. F Pediatr 1944;25:306-10.

2 Kawasaki T. Acute febrile mucocutaneous syndrome with lymphoid involvement with specific desquamation of the fingers and toes in children. Fapanese fournal of Allergy 1967;16:178-222.

${ }^{3}$ Melish ME. Kawasaki syndrome (the mucocutaneous lymph node syndrome). Annu Rev Med 1982;33:569-85.

4 Kato H, Koike S. Clinicopathologic studies of diseases associated with vasculitis-Kawasaki disease (MCLS) and infantile polyarteritis. [In Japanese.] Nippon Rinsho 1978;36:753-67.

${ }^{5}$ Kato H, Kioke S, Yokoyama T. Kawasaki disease: effect of treatment in coronary artery involvement. Pediatrics $1979 ; 63: 175-9$. 
${ }^{6}$ Kato H, Koike S, Tanaka C, et al. Coronary heart disease in children with Kawasaki disease. Fpn Circ f 1979;43:469-75.

7 Fujiwara H, Hamashima Y. Pathology of the heart in Kawasaki disease. Pediatrics 1978;61:100-7.

${ }^{\diamond}$ Bell DM, Morens DM, Holman RC, Hurwitz ES, Hunter MK. Kawasaki syndrome in the United States. 1976-1980. Am f Dis Child 1983;137: $211-4$.

${ }^{9}$ Kato S, Kimura M, Tsuji K, et al. HLA antigens in Kawasaki disease. Pediatrics 1978;61:252-5.

10 Miyamoto T, Oshima S, Domae A, et al. Allergenic potency of different house dusts in relation to contained mites. Ann Allergy 1970;28:405-12.

${ }^{11}$ Furusho K, Ohba T, Soeda T, Kimoto K, Okabe T, Hirota T. Possible role for mite antigen in Kawasaki disease. Lancet 1981 ;ii:194-5.

12 Fujimoto $T$, Kato $H$, Ichiose E, Sasaguri Y. Immune complex and mite antigen in Kawasaki disease. Lancet 1982;ii :980-1.

${ }^{13}$ Hamashima Y, Tasaka K, Hoshino T, et al. Mite-associated particles in Kawasaki disease. Lancet $1982 ;$ ii :266.

14 Hamashima Y, Kishi K, Tasaka K. Rickettsia-like bodies in infantile acute febrile mucocutaneous lymph-node syndrome. Lancet 1973;ii:42.

15 Carter RF, Haynes ME, Morton J. Rickettsia-like bodies and splenitis in Kawasaki disease. Lancet 1976;ii:1254-5.

16 Patriarca PA, Rogers MF, Morens DM, et al. Kawasaki syndrome : association with the application of rug shampoo. Lancet 1982;ii:578-80.

17 Hicks RV, Melish ME. Kawasaki syndrome: rheumatic complaints and analysis of salicylate therapy. [Abstract.] Arthritis Rheum 1979;22:621.

18 Jacobs JC. Successful treatment of Kawasaki syndrome with high-dose aspirin. [Abstract.] Pediatr Res 1978;12:494.

${ }^{19}$ Yamada K, Shinkai A, Shirahata A, Meguro T. Studies on the platelet function of acute febrile mucocutaneous lymph node syndrome and trial of prevention of thrombosis by flurbiprofen. Acta Pediatrica faponica $1977 ; 81: 1263$.

20 Hidaka $T$, Nakano $M$, Ueta $T$, Komatsu $Y$, Yamamoto $M$. Increased synthesis of thromboxane $A_{2}$ by platelets from patients with Kawasaki disease. $\mathcal{F}$ Pediatr 1983;102:94-6.

21 Butkus A, Skrinska VA, Schumacher OP. Thromboxane production and platelet aggregation in diabetic subjects with clinical complications. Thromb Res 1980;19:211-23.

${ }^{22}$ Moncada S, Vane JR. Arachidonic acid metabolites and the interactions between platelets and blood-vessel walls. $N$ Engl F Med 1979;300:1142-7.

23 Sasaguri Y, Kato H. Regression of aneurysms in Kawasaki disease: a pathological study. 7 Pediatr 1982;100:225-31.

\section{Hyperventilation as a cause of panic attacks}

The syndrome characterised by repeated panic attacks has been known by several names, including muscular exhaustion of the heart, neurasthenia, irritable heart, anxiety neurosis, effort syndrome, and cardiac neurosis. ${ }^{1}$ More recently The Diagnostic and Statistical Manual of Mental Disorders has created a subcategory of panic disorder as a diagnostic entity of its own, distinguished from generalised anxiety disorder. ${ }^{2}$ The manual's definition of panic disorder states that attacks are manifested by the sudden onset of intense apprehension, fear, or terror, often associated with feelings of impending doom. The most common symptoms experienced during an attack are dyspnoea; palpitations; chest pain or discomfort; choking or smothering sensations; dizziness, vertigo, or unsteady feelings; feelings of unreality; paraesthesias; hot and cold flushes; sweating; faintness; trembling or shaking; and fear of dying, going crazy, or doing something uncontrolled during the attack. Attacks usually last minutes; more rarely hours.

Several suggestions have been made recently for distinguishing between generalised anxiety and panic disorder. Double blind studies have shown that antidepressants are effective in the treatment of patients with panic disorders, ${ }^{3-6}$ leading to the hypothesis that panic attacks of sudden onset with appreciable autonomic symptoms and overwhelming fear may represent an "endogenous" or "biogenic" form of anxiety disorder. The mechanism by which antidepressants achieve this effect is in dispute, however, being seen by some as evidence of a central, noradrenergic basis to anxiety ${ }^{7}$ and by others as an effect on depressive features which may accompany the panic disorder. ${ }^{8}$

A second aetiological hypothesis proposes that panic attacks may be a consequence of peripheral beta adrenergic hyperactivity $^{9}$ or of a hyperdynamic beta adrenergic circulatory state. ${ }^{10}$ Beta adrenergic agonists induce panic attacks in patients already susceptible to the disorder; conversely, beta adrenergic antagonists (for example, propranolol) can reverse or suppress panic attacks. These findings have been taken to suggest that subjects who experience panic attacks may have an underlying beta adrenergic supersensitivity.

Another possible cause of panic attacks is hyperventilation, which is an important component of panic attacks but has received no mention in recent reviews. ${ }^{112}$ In contrast to the other possible causes of these attacks hyperventilation may be diagnosed with simple tests and can be treated.

Lewis suggested that many, if not all, people may sometimes react to stress or anxiety by overbreathing and that this might be part of the "fight or flight" response. ${ }^{13}$ In the absence of a compensating increase in metabolic rate, hyperventilation lowers the partial pressure of carbon dioxide in arterial blood $\left(\mathrm{PaCO}_{2}\right)$ and increases its $\mathrm{pH}$. These two changes are thought to cause the wide range of symptoms-dizziness, feelings of unreality, paraesthesias, tremor, choking, chest pain, and palpitations-which closely resemble those generally accepted as typical of panic attacks. The unpleasant sensations that occur after a fall in $\mathrm{PaCO}_{2}$ may make the patient concerned about his health and may provoke specific fears of heart attack or sudden death. Increased apprehension may then lead to further hyperventilation and a vicious circle of increasing symptoms. ${ }^{13}$ Clark's review ${ }^{14}$ catalogued the evidence that patients with anxiety neuroses hyperventilate when compared with controls ${ }^{15}$; that hyperventilation may cause anxiety ${ }^{16} ;$ and that controlled ventilation improves the symptoms of some anxious patients. ${ }^{17-20}$ Hyperventilation has also been implicated in agoraphobia, ${ }^{21}$ in which the symptoms of panic resemble those in generalised anxiety disorders. ${ }^{22}$

Usually the diagnosis of hyperventilation depends on assessing the effects of a provocation test, in which the patient voluntarily overbreathes for two to three minutes. The symptoms experienced are compared with those experienced by that same patient in a typical panic attack. ${ }^{17}$ Patients who recognise the similarity of the symptoms may be assumed to hyperventilate during their panic attacks; patients who do not recognise the symptoms may be assumed not to hyperventilate. Once diagnosed, treatment consists of training in responding to stress or anxiety with slow breathing. ${ }^{19} 20$

Lum has claimed that $95 \%$ of a sample of over 1000 patients diagnosed as hyperventilating by this method lost all their symptoms or were appreciably improved by a treatment programme which included training in slow breathing. ${ }^{17}$ Clearly claims for the efficacy of this treatment and for the effectiveness of this diagnostic technique need to be tested in a controlled trial with objective measures.

Panic attacks may be precipitated by the same circumstances as cause hyperventilation. Both panic attacks and hyperventilation commonly occur after muscular exertion, ${ }^{1323-25}$ and both may occur while falling asleep or waking. ${ }^{132}$ Hyperventilation may be either a component of the initial event, as in exertion, or a response to it, as perceiving a threat. The maintenance of the disorder may result from the development of a fear of any bodily sensation resembling those experienced during the initial panic attack in a feedback loop, as described by Breggin. ${ }^{26}$ This mechanism postulates particular somatic 